\title{
NOUVELLE
}

\section{La lactadhérine, nouvel acteur de l'angiogenèse}

Jean-Sébastien Silvestre, Clotilde Théry, Bernard Lévy, Alain Tedgui, Sebastian Amigorena, Ziad Mallat
J.S. Silvestre, B. Lévy, A. Tedgui, Z. Mallat : Centre de Recherche Cardiovasculaire, Inserm U.689, Hôpital Lariboisière, 41, boulevard de la Chapelle, 75475 Paris Cedex 10, France. C. Théry, S. Amigorena : Inserm U.653, Institut Curie, 26, rue d'Ulm, 75005 Paris, France. mallat@larib.inserm.fr
> Le VEGF (vascular endothelial growth factor) est un facteur de croissance majeur pour le développement des nouveaux vaisseaux sanguins. II agit principalement par l'intermédiaire de son récepteur, le VEGF-R2 (KDR/FIk1 ), en activant de nombreuses voies de signalisation, notamment celles dépendantes des intégrines $\alpha v \beta 3$ et $\alpha v \beta 5$.

La lactadhérine est une glycoprotéine sécrétée par les glandes mammaires. Son rôle physiologique reste obscur. Elle est impliquée dans la reconnaissance des cellules apoptotiques par les macrophages [1,2], elle présente des homologies de séquence avec une protéine pro-angiogénique Del-l et possède une séquence RGD lui permettant de se lier aux intégrines $\alpha v \beta 3$ et $\alpha v \beta 5$ $[3,4]$. L'ensemble de ces propriétés pouvait donc conférer à la lactadhérine un potentiel pro-angiogénique.

Pour étayer cette hypothèse, nous avons analysé, dans un premier temps, l'expression de la lactadhérine dans le tissu vasculaire. L'ARN et la protéine ont été détectés dans l'aorte murine et humaine, soulignant son rôle éventuel dans le développement de nouveaux vaisseaux (Figure IA).

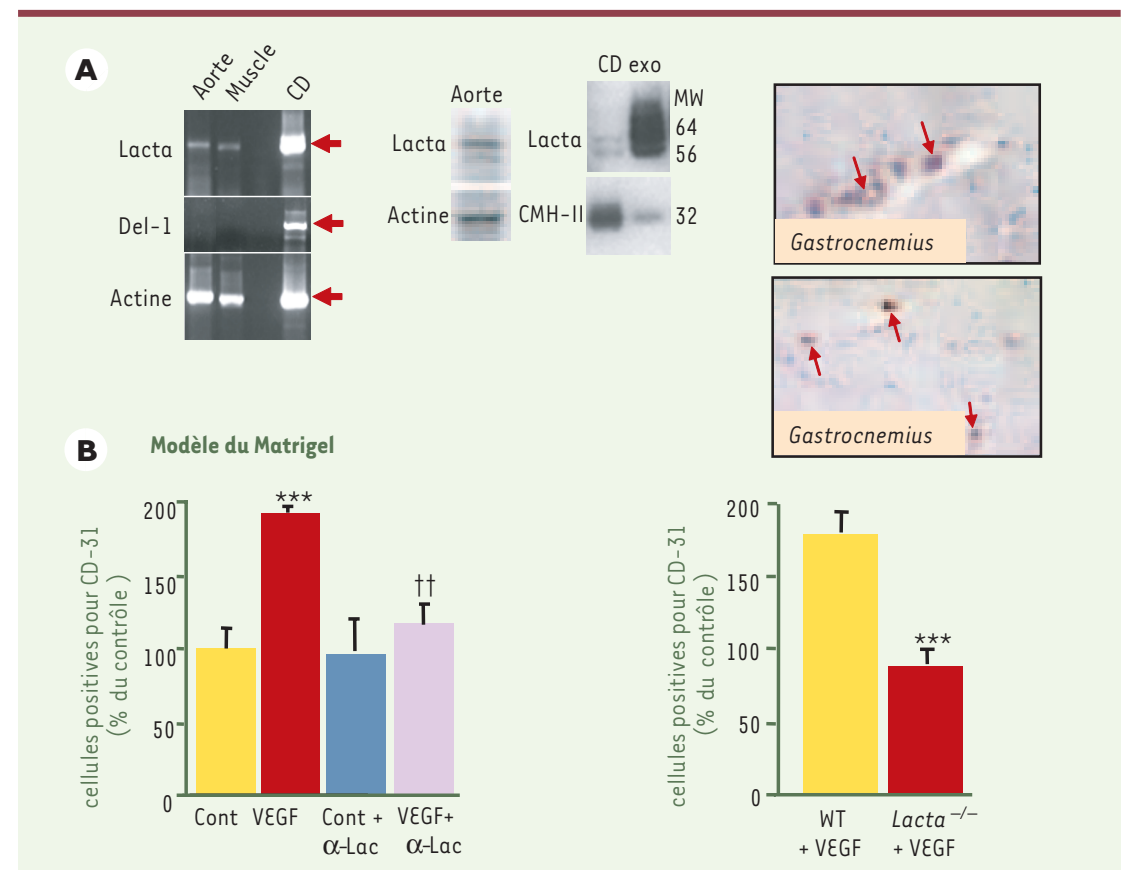

C Modèle d'ischémie du membre inférieur

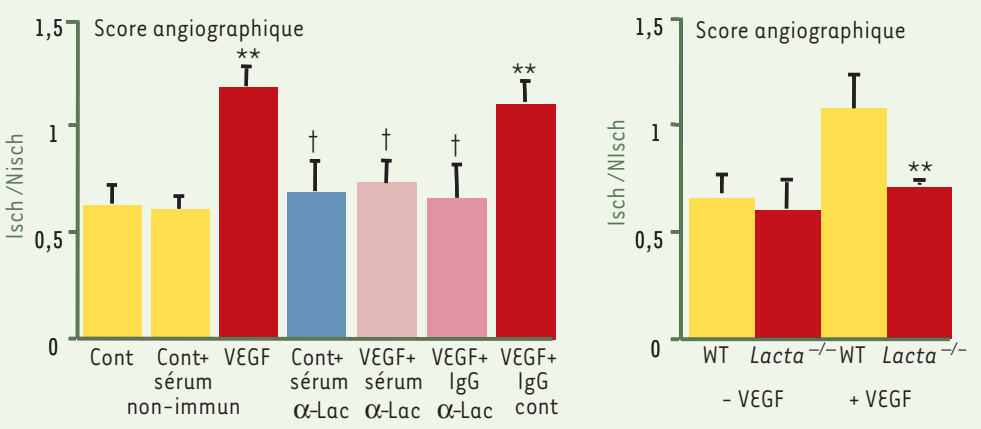

Figure 1. Rôle de la lactadhérine dans le processus de néovascularisation. A. À gauche: PCR représentative du niveau d'expression de la lactadhérine et de son homologue Del-1 dans l'aorte de souris, le muscle squelettique (muscle) et les cellules dendritiques (CD, témoin positif). Au milieu : Western blot représentatif de la quantité de lactadhérine dans l'aorte de souris (exo: exosomes qui expriment un fort taux de lactadhérine). À droite : images représentatives de coupes de muscle gastrocnemius de souris hybridées avec un anticorps dirigé contre la lactadhérine, montrant une expression vasculaire et interstitielle de la lactadhérine. B. Quantification du nombre de cellules exprimant le marqueur de cellules endothéliales CD31 dans le Matrigel traité ou non avec du VEGF et injecté dans le dos de souris témoins (traitées ou pas par un anticorps neutralisant dirigé contre la lactadhérine, $\alpha$-Lac) ou de souris déficientes en lactadhérine ( Lacta $^{-/-}$). ${ }^{\star \star \star} p<0,01$ versus témoin ; ${ }^{\dagger \dagger} p<0,01$ versus VEGF. C. À gauche, évaluation de la densité vasculaire, 21 jours après l'ischémie, dans le membre inférieur de souris traitées ou non avec du VEGF et un sérum dirigé contre la lactadhérine (sérum $\alpha$-Lac), des IgG purifiées dirigées contre la lactadhérine (IgG $\alpha$-Lac) ou des IgG témoins (IgG cont, dirigées contre la thrombospondine-1, un marqueur des cellules endothéliales). À droite, évaluation de la densité vasculaire, 21 jours après l'ischémie, dans le membre inférieur de souris témoins (WT) ou déficientes en lactadhérine (lacta ${ }^{-/-}$) traitées ou non avec du VEGF. $* \star<p<0,01 ;{ }^{\dagger} p<0,05$. 
Nous avons alors développé un certain nombres d'outils (anticorps neutralisants dirigés contre les formes humaine et murine de la lactadhérine, protéine recombinante)
A

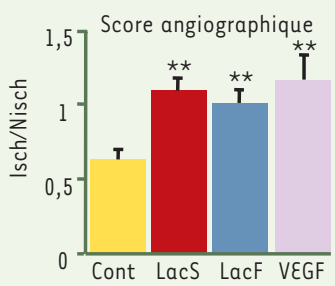

\section{C}

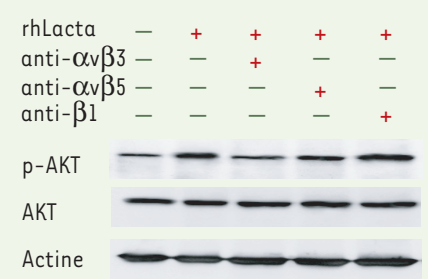

D

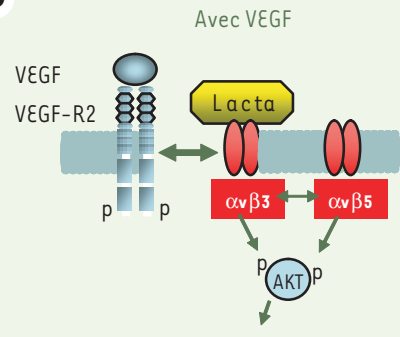

Phénotype angiogénique et avons créé une lignée de souris déficientes pour la lactadhérine, afin d'analyser l'implication de cette molécule dans le développement des néovaisseaux.

3
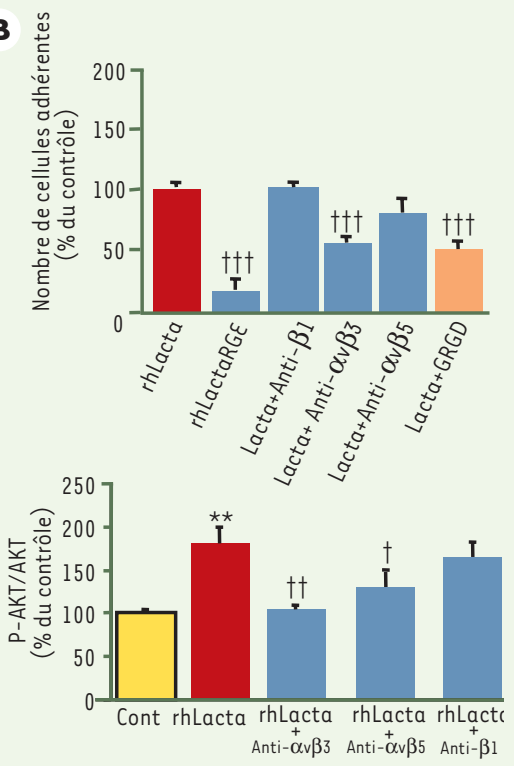

Sans VEGF

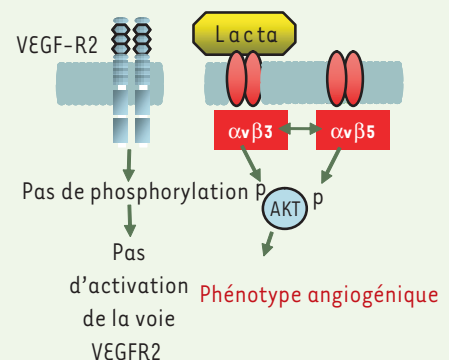

Figure 2. La lactadhérine interagit avec l'intégrine $\alpha v \beta 3$ pour activer le processus de néovascularisation. A. Évaluation de la densité vasculaire, 21 jours après l'ischémie, dans le membre inférieur de souris témoins traitées ou non avec un plasmide vide (Cont) ou codant pour la forme courte de la lactadérine (Lac S), sa forme longue (Lac F) ou le VEGF (VEGF). ${ }^{\star *} p<0,01$ versus témoin. $\boldsymbol{B}$. Quantification du nombre de cellules endothéliales humaines (HUVEC) qui adhèrent sur la lactadhérine (rhLacta) ou sur une forme mutée de la lactadhérine (rhlactaRGE) en présence ou en l'absence d'un anticorps neutralisant dirigé contre l'intégrine $\beta 1$ (Lacta + Anti $\beta 1$ ), l'intégrine $\alpha v \beta 3$ (Lacta + Anti $\alpha v \beta 3)$ ou l'intégrine $\alpha v \beta 5$ (Lacta + Anti $\alpha v \beta 5)$, et, enfin, en présence du peptide GRGD (Lacta+GRGD). ${ }^{\dagger \dagger} p<0,001$ versus rhLacta. $C$. Images représentatives et quantification du niveau de phospho-AKT et d'AKT dans des cellules endothéliales humaines (HUVEC) traitées ou non avec de la protéine recombinante lactadhérine (rhLacta) et un anticorps neutralisant dirigé contre l'intégrine $\beta 1$ (rhLacta+Anti $\beta 1$ ), l'intégrine $\alpha v \beta 3$ (rhLacta+Anti $\alpha v \beta 3$ ) ou l'intégrine $\alpha v \beta 5$ (Lacta+rhAnti $\alpha v \beta 5) .{ }^{* \star} p<0,01$ versus témoins; ${ }^{\dagger} p<0,05 ;{ }^{\dagger} p<0,01$ versus rhLacta. D. Mécanisme d'action de la lactadhérine. En présence de VEGF, la phosphorylation de son récepteur, le VEGF-R2, permet la formation d'un complexe trimoléculaire VEGF-R2/lactadhérine/intégrine $\alpha v \beta 3$ qui initie le processus angiogénique. En l'absence de VEGF, la lactadhérine, en interagissant directement avec l'intégrine $\alpha v \beta 3$, est capable d'initier le développement de nouveaux vaisseaux sanguins.
Rôle de la lactadhérine dans l'angiogenèse induite par le VEGF

Dans un premier temps, son potentiel proangiogénique a été testé dans un modèle d'angiogenèse in vivo, le Matrigel. Le VEGF induit une forte infiltration de cellules, essentiellement de type endothélial, dans le Matrigel. Nous avons montré que l'utilisation d'un anticorps anti-lactadhérine ou de souris déficientes en lactadhérine atténue totalement l'effet pro-angiogénique du VEGF (Figure 1B).

\section{Rôle de la lactadhérine dans la néovascularisation post-ischémique induite par le VEGF}

Nous avons également évalué le rôle de la lactadhérine dans un modèle pathologique d'ischémie du membre inférieur chez la souris. Dans ce contexte également, l'utilisation d'un anticorps antilactadhérine ou de souris déficientes en lactadhérine démontre que l'activation du processus de néovascularisation post-ischémique, par le VEGF, requiert la présence de lactadhérine (Figure 1C).

\section{Mécanisme d'action de la lactadhérine} Les effets chimiotactiques et mitogéniques du VEGF sont classiquement médiés par les protéine kinases ERK (extracellular signal-related kinase) et AKT (sérine/thréonine kinase). Dans le muscle ischémié comme dans des cultures de cellules endothéliales humaines, il apparaît que l'effet du VEGF sur la phosphorylation d'AKT est bloqué par l'inhibition ou l'absence de lactadhérine.

\section{Stratégie thérapeutique fondée sur la surexpression de lactadhérine} La lactadhérine étant nécessaire à l'effet du VEGF, nous avons considéré que cette molécule pourrait avoir un effet thérapeutique dans le traitement des maladies ischémiques. Nous avons développé des plasmides codant pour la forme longue ou courte de la lactadhérine et démontré que l'administration, par la méthode d'électro-transfert in vivo, de lacta- 
dhérine, en l'absence de VEGF exogène, induit une forte néovascularisation dans le membre inférieur ischémié des souris traitées (Figure 2A). Cet effet semble lié à l'activation des intégrines $\alpha v \beta 3$ et $\alpha v \beta 5$. En effet, l'adhérence de cellules endothéliales à des plaques de culture recouvertes de lactadhérine est bloquée par un anticorps anti- $\alpha v \beta 3$ (Figure 2B). De plus, l'administration de la protéine recombinante lactadhérine augmente, en l'absence de VEGF, la phosphorylation d'AKT dans des cellules endothéliales en culture. Or, cet effet est diminué par l'utilisation d'un anticorps anti$\alpha v \beta 3$ ou anti- $\alpha v \beta 5$, démontrant ainsi que la lactadhérine interagit avec les intégrines $\alpha v \beta 3$ et $\alpha v \beta 5$ pour activer la protéine kinase AKT, et donc le processus angiogénique (Figure 2C).

\section{Discussion}

Ce travail démontre pour la première fois que la lactadhérine est exprimée dans les cellules vasculaires et joue un rôle clé dans la phosphorylation de la protéine kinase AKT par le VEGF, et par conséquent dans l'effet de ce facteur de croissance sur le processus de néovascularisation [5]. L'effet du VEGF par son récepteur VEGFR2 requiert I'association de ce dernier avec l'intégrine $\alpha v \beta 3$ et l'inhibition de $\alpha v \beta 3$ diminue l'effet pro-angiogénique du VEGF [6]. Nos résultats démontrent que la lactadhérine est un facteur endogène qui, en interagissant avec l'intégrine $\alpha v \beta 3$, module la voie de signalisation dépendante de VEGF/VEGFR2. L'activation des intégrines, même en l'absence de facteurs de croissance, peut affecter l'angiogenèse. Cela a notamment été démontré pour Del1, l'analogue de la lactadhérine [3, 4]. De même, nos travaux prouvent que la lactadhérine interagit avec les intégrines $\alpha v \beta 3$ et $\alpha v \beta 5$ et, par un mécanisme dépendant d'AKT, qu'elle est capable d'activer le développement de nouveaux vaisseaux dans un contexte ischémique en l'absence de
VEGF exogène (Figure 2D). Ainsi, une stratégie thérapeutique fondée sur la surexpression de lactadhérine pourraît être proposée dans le traitement des pathologies ischémiques. $\diamond$

Lactadherin promotes VEGF-dependent neovascularization

\section{RÉFÉRENCES}

1. Hanayama R, Tanaka M, Miwa K, et al. Identification of a factor that links apoptotic cells to phagocytes. Nature $2002 ; 417$ : 182-7.

2. Hanayama R, Tanaka M, Miyasaka K, et al. Autoimmune disease and impaired uptake of apoptotic cells in MFG-\&8-deficient mice. Science $2004 ; 304$ : 1147-50.

3. Ho HK, Jang JJ, Kaji S, Spektor G, et al. Developmental endothelial locus-1 (Del-1), a novel angiogenic protein: its role in ischemia. Circulation $2004 ; 109$ : 1314-9.

4. Zhong J, Eliceiri B, Stupack D, et al. Neovascularization of ischemic tissues by gene delivery of the extracellular matrix protein Del-1. J Clin Invest $2003 ; 112: 30-41$.

5. Silvestre JS, Théry C, Hamard G, et al. Lactadherin/MFG-\&8 promotes VEGF-dependent neovascularization. Nat Med $2005 ; 11:$ 499-506.

6. Borges $\varepsilon$, Jan $Y$, Ruoslahti $\varepsilon$. Platelet-derived growth factor receptor beta and vascular endothelial growth factor receptor 2 bind to the beta 3 integrin through its extracellular domain. J Biol Chem $2000 ; 275$ : 39867-73.

\section{NOUVELLe}

\section{Cryptococcus neoformans change de capsule après la traversée de la barrière hémato-encéphalique}

Caroline Charlier, Fabrice Chrétien, Olivier Lortholary, Françoise Dromer

> Cryptococcus neoformans est l'agent de la cryptococcose, une infection opportuniste affectant les malades atteints du sida ou d'autres déficits de l'immunité cellulaire. Elle réalise une infection disséminée, dont la localisation la plus fréquente et la plus grave est une méningo-encéphalite, mortelle dans environ $20 \%$ des cas malgré un traitement antifongique adapté [1].
Alors que l'incidence de la maladie a diminué dans les pays occidentaux avec l'ère de la trithérapie, la cryptococcose reste une cause majeure de méningite en Afrique subsaharienne [2]. La physiopathologie de l'infection reste mal comprise. La porte d'entrée est probablement respiratoire, aboutissant

\author{
C. Charlier, F. Dromer : Unité de Mycologie moléculaire, CNRS \\ FRE 2849, Institut Pasteur, 28, rue du Docteur Roux, \\ 75015 Paris, France. \\ F. Chrétien : Inserm EMI 0011, Université Paris 12, Faculté de \\ Médecine de Créteil et Département de Pathologie, Hôpital \\ Henri Mondor, 51, avenue du Maréchal de Lattre de Tassigny, \\ 94010 Créteil, France. \\ 0. Lortholary: Unité de Mycologie moléculaire, CNRS FRE 2849, \\ Institut Pasteur, 28, rue du Docteur Roux, 75015 Paris, France \\ et Service de Maladies Infectieuses et Tropicales, Université \\ Paris V, Hôpital Necker-Enfants malades, 149, rue de Sèvres, \\ 75743 Paris Cedex 15 France. \\ olivier.lortholary@nck.ap-hop-paris.fr
}

à une primo-infection pulmonaire souvent asymptomatique, puis la levure persisterait dans l'organisme sous une forme latente [3]. À la faveur d'une immunosuppression, la levure serait 